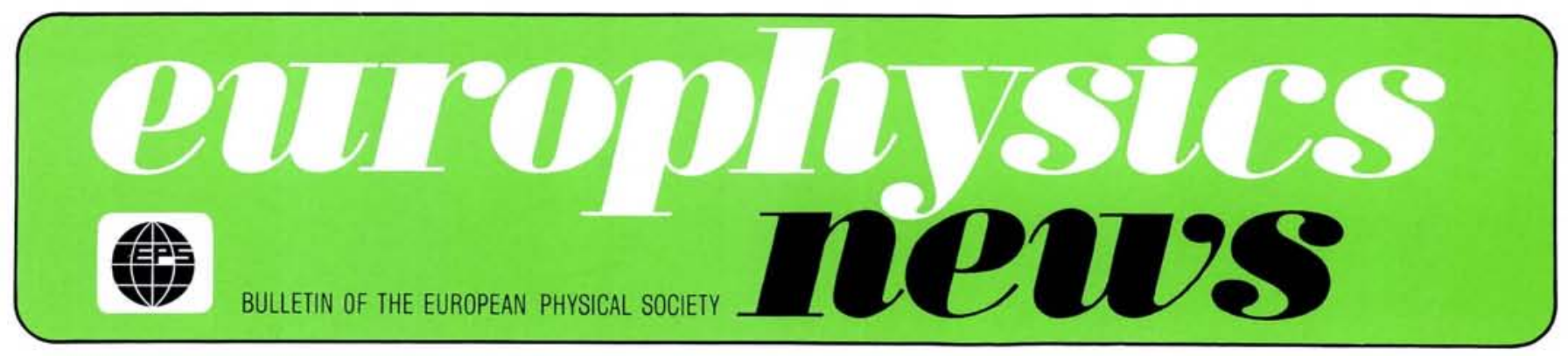

J.A.

February 1982

\title{
Self-Organization of Turbulence
}

\section{Akira Hasegawa, Murray Hill, New Jersey}

\author{
(Bell Laboratories)
}

\author{
A brief review of self-organization \\ with particular reference to hydrodynamic and magnetohydrodynamic turbulence
}

Theoretical treatments of turbulence in fluids and plasmas often assume that the turbulence is isotropic and homogeneous. It is also often considered that turbulence produces uniformly distributed chaos, even when starting with a coherent initial condition. Recently, however, phenomena which do not obey these classic concepts have emerged. For example, in two-dimensional Navier-Stokes turbulence, an organized flow or structure is found to appear even from a chaotic initial condition. This manuscript attempts to review some of the recent developments of a phenomenon called self-organization in the field of hydrodynamics and plasma physics.

Two-dimensional Navier-Stokes Turbulence

"Self-Organization" is a process by which an ordered structure appears from initial uniformly distributed turbulence. To introduce the concept of self-organization, let us look at the classic example of twodimensional Navier-Stokes turbulence. In 1949 , Onsager pointed out that two-dimensional Navier-Stokes turbulence could have a negative temperature state as a consequence of the existence of two conserved quantities. They are the energy and a quantity called enstrophy that is the squared vorticity. We note that in three-dimensional Navier-Stokes turbulence, only energy is conserved. If one constructs the Gibbs distribution using these two conserved quantities, the mean squared velocity field (the average energy) can have a negative value in two-dimensional Navier-Stokes turbulence. This fact is related to the formation of self-organization.

In 1967, Kreichman showed that the energy spectrum in two-dimensional Navier-Stokes turbulence, cascades to longer wave-lengths and the enstrophy spectrum cascades to a shorter wavelength regime. Consequently, the energy tends to accumulate at the longest wavelength that the system can accept. When this occurs, the flow pattern of the fluid becomes ordered. The loss of entropy which results from the appearance of ordered structure in the velocity field is compensated for by an increase of entropy in the vortex field. Here it is important to recognize that such a process is not the consequence of a simple linear filtering of the high wavenumber modes. Since the dissipation in NavierStokes turbulence is viscous, it operates on the higher wavenumber modes more strongly than on the lower wavenumber modes, the higher wavenumber portion of a uniformly distributed spectrum can be linearly filtered. This can lead to a larger energy concentration in the longer wavelength regime. However, the self-organization is not a consequence of such a linear process, but is a process in which the nonlinear mode couplings carry energy to long wavelengths; therefore, the time scale which produces the self-organized state is much shorter than the linear or dissipation time scale.

It is possible to obtain the velocity field, $v$, which is expected to appear as the consequence of self-organization. Since the enstrophy dissipates while the energy remains constant, the self-organized state can be described by a variation of minimization of enstrophy $\int(\nabla \times v)^{2} d V$ with a constraint that the energy $\int v^{2} d V$ remains constant,

$$
\delta \int(\nabla \times v)^{2} d V-\lambda \delta \int v^{2} d V=0,
$$
where $\lambda$ is the Lagrange multiplier. This variation gives a differential equation for the velocity field,

$$
\nabla \times \nabla \times v-\lambda v=0 .
$$

This equation can be easily solved for given two-dimensional boundary conditions, the solution giving the largest vortex pattern satisfying these conditions. The solution for a smallest value of $\lambda$, which corresponds to the minimum enstrophy field, is the largest vortex structure that fits the boundary conditions. Bretherton and Haidvogel in 1976 obtained a self-organized state of Rossby wave turbulence using the variational principle.

It is interesting to note that although the original equation is nonlinear and insoluble, the equation that describes the self-organized state is linear and soluble. This is analogous to the soliton problem where the soliton producing equation can be solved by means of linear equations. I will discuss the relation between the soliton and the self-organized state later.

As can be seen from the example of 2-D Navier-Stokes turbulence, self-organization exists in a system with two or more constants of motion, and in addition, a selective dissipation among these con-

$\begin{array}{lr}\text { Contents } & \\ \text { Self-Organization of Turbulence } & 1 \\ \text { EPS Organized Conferences } & 4 \\ \text { 1981 Nobel Prizes in Physics } & \\ \text { Kai Siegbahn } & \\ \text { Nicolaas Bloembergen \& } & 5 \\ \text { Arthur Schawlow } & 6 \\ \text { Teaching Abroad } & 7 \\ \text { IX-Summer School on Quantum Optics } & 8 \\ \text { Physics of Intercalation Compounds } & 8 \\ \text { Hollow Cathode Laser Research } & 9 \\ \text { Physics Periodicals in Europe } & 11 \\ \text { New EPS Individual Members } & 11 \\ \text { Society News } & 12\end{array}$



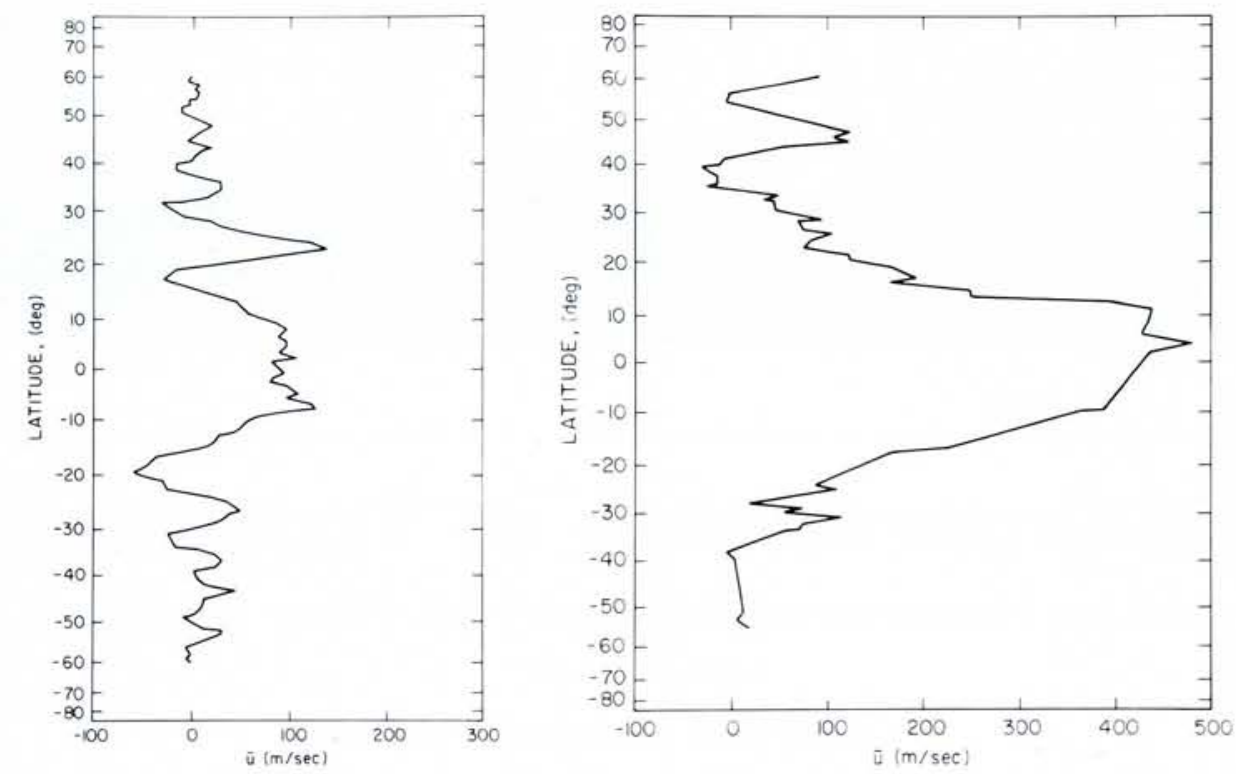

Fig. 1a (Left) - Profile of Jupiter's mean eastward wind measured relative to Jupiter's rotations (result of Voyager 1 measurement).

Fig. $1 b$ (Right) - Profile of Saturn's mean eastward wind measured relative to Saturn's rotation (result of Voyager 1 measurement).

stants. In the above example, the enstrophy and energy are the two constants and the selective dissipation affects the enstrophy.

\section{Magnetohydrodynamic Turbulence}

In magnetohydrodynamic turbulence in which the kinetic and pressure energies are relatively small compared to the magnetic field energy, there also exist two constants of motion: the magnetic field energy and the magnetic helicity, $\int \mathbf{A} \cdot \mathbf{B d V}$ ( $\mathbf{B}$ and $\mathbf{A}$ are the magnetic flux density and its vector potential). Here it can be shown that the energy selectively dissipates because the magnetic helicity has a weaker wave number dependency than the energy. Therefore, using a similar variational method, one can obtain the field equation for the self-organized state

$$
\delta \int \mathbf{B}^{2} \mathbf{d} \mathbf{V}-\lambda \delta \int \mathbf{A} \cdot \mathbf{B d} \mathbf{V} \text {. }
$$

This equation leads to a partial differential equation of the form

$$
\nabla \times \mathbf{B}-\lambda \mathbf{B}=0 .
$$

This shows that the current, $J$, is parallel to the magnetic field. Such a configuration is called force-free because the $\mathbf{J} \times \mathbf{B}$ force is zero and has been derived by Woltjer and later by Taylor. In particular, Taylor proposed that the so-called reverse field equilibrium of Zeta pinch is the consequence of a plasma achieving its minimum energy state. We note, that in self-organization the system does not necessarily reach the minimum energy state, as is seen in the example of two-dimensional Navier-Stokes turbulence. At present, a number of experiments indicate that the plasma goes through a violent turbulent state to reach a state with the field structure described by Eq. (4).
Rossby Wave; Drift Wave Turbulence

In 1978, Hasegawa and Mima derived a model field equation for the electrostatic potential which describes the drift wave turbulence in a magnetized plasma. The structure of the equation is analogous to the Rossby wave equation which describes the velocity field on the surface of a rotating planet. The structure of the equation is

$$
\begin{aligned}
& \frac{\partial}{\partial t}\left(\nabla^{2} \phi-\phi\right)- \\
& \quad[(\nabla \phi \times \hat{z}) \cdot \nabla]\left[\nabla^{2} \phi-\ln \left(n_{0}\right)\right]=0,
\end{aligned}
$$

where $n_{0}$ is the plasma density. Here $\nabla$ is a two-dimensional operator perpendicular to the magnetic field $(\bar{z})$. This equation is similar to the two-dimensional NavierStokes equation except for the inhomogeneous term, $\ln \left(n_{0}\right)$ which arises from the inhomogeneity in the plasma density. Because of this inhomogeneous term, this equation admits a linear wave called the drift wave. (In the atmosphere this is called the Rossby wave and exists due to the inhomogeneity in the Coriolis parameter.) The equation also has two conserved quantities, energy and enstrophy. However, in the regime of weak nonlinearity as compared with the linear wave frequency, the wave quantum is also conserved. These three conserved quantities play a very interesting role in the turbulence described by Eq. (5).

In a strongly nonlinear regime in which the nonlinear term overrides the linear term, the energy cascades to longer wavelengths and the enstrophy cascades to shorter wavelengths as in the case of the two-dimensional Navier-Stokes turbulence. As the energy is cascaded to longer wavelengths thereby, the nonlinear term becomes increasingly weaker compared to the linear term, the wave nature becomes manifested. That is, the wave frequency can become larger than the nonlinear frequency shift decided by the nonlinear mode coupling term. When this happens the conservation of the wave quantum becomes important and the energy now tends to cascade to smaller frequencies rather than smaller wavenumbers. The frequency is proportional to the wavenumber in the direction perpendicular to the inhomogeneity. In the case of the drift wave, it corresponds to the wavenumber in the direction perpendicular to the density gradient, while in the case of the Rossby wave the frequency is proportional to the wavenumber in the east-west direction. This tends to produce an anisotropic spectrum because now the energy cascades only to smaller wavenumbers in these directions.

The consequence of this is the formation of zonal flow patterns in the direction perpendicular to the inhomogeneity as suspected by Rhines in 1975. In the case of plasma, periodic zonal flow appears with the flow velocity directed in the azimuthal direction while for the Rossby wave the zonal flow appears in the east-west direction with periodicity in the north-south direction. The periodicity in the northsouth direction is given by the value of wave number ${ }^{-} k_{\mathrm{c}}$ at which the spectrum condenses. Hasegawa, Kodama and Maclennan have obtained this wavenumber $k_{\mathrm{c}}$ in terms of the mean squared potential fluctuation $\left(\phi^{2}\right)$ of the turbulence.

$$
k_{\mathrm{c}} \cong\left[\kappa^{2} /\left(\phi^{2}\right)\right]^{1 / 6},
$$

where $\kappa$ is the measure of inhomogeneity scale, $\nabla \ln \left(n_{0}\right)$. Most recently Hasegawa ap- 
plied this result to the Jovian and Saturnian wind systems. Rossby wave turbulence can also be described by Eq. (5) if the inhomogeneous density term is replaced by the minus of the inhomogeneous Coriolis parameter. By extending the theory which was derived for a constant inhomogeneity (constant $\kappa$ ) in Cartesian coordinates to the non-uniform inhomogeneity which represents the Coriolis force on a planetary surface one can obtain the variation of width of the zonal flow from Eq. (6) as a function of the intensity of turbulence.

The recent Voyager mission found both Jupiter and Saturn to have zonal wind patterns with the wind speed approaching a fraction of the sound speed. By applying the above results to Jupiter and Saturn, Hasegawa obtained the width of the wind zones as a function of level of turbulence in scale height which is deduced from the observed wind speed. When the widths of the zonal flows are expressed in terms of their latitudinal angle $\theta_{c}(\theta)$, Eq. (6) becomes,

$\theta_{c}(\theta)=180^{\circ} \frac{(g H)^{1 / 3}}{(2 \sqrt{2} R \Omega)^{2 / 3}} \cdot \frac{\phi^{1 / 3}}{(\sin \theta \cos \theta)^{1 / 3}}$ (7)

where $g$ is the constant of gravity, $H$ is the scale height, $R$ is the radius of the planet, $\Omega$ the angular velocity of the planetary rotation and $\phi$ is the relative perturbation in scale height due to turbulence. Fig. 1 shows the observed value of the wind speed as a function of latitudinal angle for Jupiter (a) and Saturn (b). When the observed value of the width of each zone are plotted in comparison with the theoretical value as obtained from Eq. (7), fairly good agreements are seen (Fig. 2).

From these examples it is clear that selforganization is a process which appears as a consequence of the dissipation of lowerorder conserved quantities. Therefore, selforganization can be defined as a process in which these weakly conserved quantities dissipate at a rate faster than the linear dissipation rate. This can be proved rigorously in the examples presented here. In a computer simulation, it often takes a very long time to see the self-organized state. This is because of the remaining turbulence which still contains many higher order wave numbers. In addition, as the initially given energy dissipates, the Reynolds number is reduced, resulting in the longer time scale of the nonlinear mode coupling. Therefore self-organization might be more apparent in a driven system in which there exists a continuous input of energy to the system at the proper range of wavenumbers. The Jupiter and Saturn wind systems correspond to such a case.

Although both of these phenomena seem analogous in that some coherent object appears as a consequence of nonlinear mode coupling, there exist important physical distinctions. In self-organization, the presence of selective dissipation is essen-

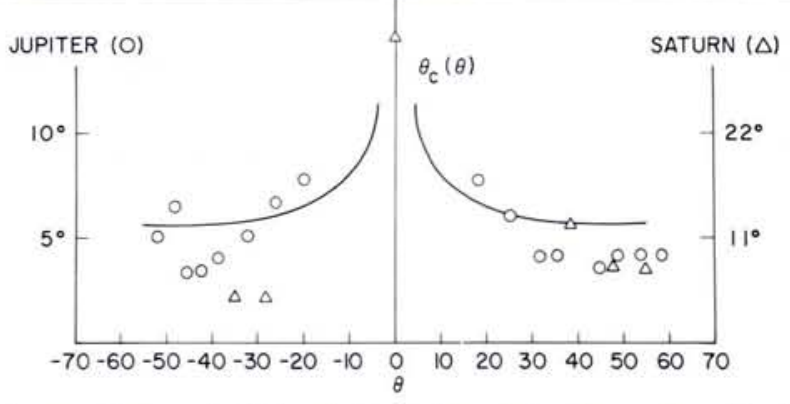

Fig. 2 - Dependence of the zonal width on latitudinal angle $\theta_{c}(\theta)$ as predicted by theory, Eq. (7), is shown by the solid curve. The observed values are plotted by the circles for Jupiter and triangles for Saturn. both eastward and westward winds are used here.

tial, while in the soliton, the absence of dissipation is essential for the complete integrability of the original equation. Hasegawa, Kodama and Watanabe have attempted to reconcile these two areas by studying the Korteweg-de Vries equation

with dissipation. They showed that such a system does have the self-organizing property in that the lower-order conserved quantities dissipate at a rate faster than the dissipation rate and that the self-organized state emerges in the form of a single soliton.

\section{NATO ADVANCED STUDY INSTITUTE
July 26-August 51982
University of Antwerp, Belgium
(Corsendonk)
(U.I.A.)}

Theory: Quantum field prerequisites. Feynman path integral formulation of electron-phonon interaction for polarons and excitons. Canonical transformations and Green's function techniques. Many body effects. Phase transitions and polarons. Droplets...

Experiment: Drift mobility. Hall-mobility. Cyclotron-resonance. Optical properties. Streaming motion and population inversion of polarons. Raman scattering and many polaron effects. Spectroscopy of excitons...

Preliminary list of lecturers:

A. Baldereschi (CH), F.C. Brown (USA), J. Devreese (B), R. Elliott (UK), R. Evrard (B), S. Komiyama (Japan), M. Lax (USA), H. Leschke (D), T. Reinecke (USA), W. Richter (D), L. Sham (USA), R. Stradling (UK), W. von der Osten (D).

Scientific Committee: J.T. Devreese (Chatrman), F. Peeters, M. Mariën.

Further information: International Advanced Study Institute

c/o Mrs. R.-M. Vandekerkhof, Department of Physics,

University of Antwerp, Universiteitsplein 1, B-2610 WILRIJK

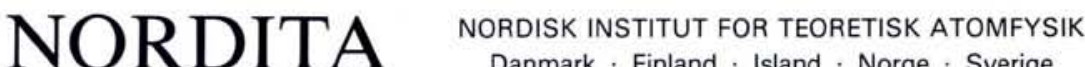

\section{Assistant Professorship in Theoretical Astrophysics}

NORDITA, the Nordic theoretical physics institute located at the Niels Bohr Institute of Copenhagen University, has an opening starting September 1982 for a theoretical astrophysicist. The initial appointment will be for three years, with the possibility of renewal up to a total of six years. Salary will be in the range of $170,000-250,000$ Danish Kroner depending on experience. The successful applicant is expected to guide fellows at roughly the post-doctoral level and to interact with colleagues at NORDITA and elsewhere in the Nordic countries. This position provides excellent opportunities to pursue original research, to travel to other institutes and to meetings, and to invite guest scientists. There is no restriction on the applicant's field of research, though preference will be given to those areas where physics and astrophysics are most closely coupled. Recent research activities at NORDITA have involved neutron stars, supernovae, X-ray sources, cosmic rays and the formation of galaxies.

Send before 15 March, a curriculum vitae, list of publications (with titles) and the names of at least three referees to

$$
\begin{aligned}
& \text { N.R. Nilsson, NORDITA, Blegdamsvej } 17 \\
& \text { DK-2100 Copenhagen O, Denmark }
\end{aligned}
$$

There is no restriction as to the sex or nationality of the applicant. 